\title{
Reflexiones sobre la formación en Psicología Clínica: el camino hacia la Pericia
}

\section{Reflections on Clinical Psychology Training: the road to Expertise}

\author{
Sergio Revenga Montejano ${ }^{(1)}$ y Ana Martín García ${ }^{(2)}$ \\ (1) Hospital de Guadalajara, España \\ (2) Sanatorio Esquerdo, España
}

\begin{abstract}
Resumen: La eficacia de la formación en Psicología Clínica tiende a darse por supuesta más que puesta a prueba. Por este motivo algunos autores han comenzado a preocuparse por esta cuestión concluyendo que su eficacia no está garantizada (Malouff, 2012; Ford, 1979). En el presente trabajo se revisa la investigación en torno a los factores del psicoterapeuta que se relacionan con mejores resultados en terapia y los mecanismos para el logro de la pericia o excelencia en la labor clínica (e.g., Hill, Spiegel, Hoffman, Kivlighan y Gelso, 2017) tales como la supervisión, la práctica deliberada o el desempeño relacional - contrastando estos planteamientos con la experiencia en la actual formación como psicólogos vía PIR y reflexionando acerca de las implicaciones que tendría asumir un enfoque basado en la pericia tanto para pacientes y profesionales como para la propia institución.
\end{abstract}

Palabras clave: Psicología Clínica, formación, Pericia, Psicólogo Interno Residente, Práctica Deliberada, Supervisión

\begin{abstract}
The efficacy of Clinical Psychology training is usually taken for granted rather than being tested. This is why some authors have begun to take care of this matter, concluding that its effectiveness is not guaranteed (Maluff, 2012; Ford, 1979). In the present work, research about therapist factors that are related with better outcomes and the process of reaching expertise in the clinical practice are examined (e.g., Hill, Spiegel, Hoffman, Kivlighan y Gelso, 2017) - such as supervision, deliberate practice or relational skills - contrasting this approaches with the actual PIR training program of Clinical Psychology specialists, reflecting on the implications of assuming this approach for patients and professionals and also for the institution.

Key words: Clinical Psychology, training, Expertise, Clinical Psychology Intern, Deliberate Practice, Supervision
\end{abstract}

Sergio Revenga Montejano es Psicólogo Clínico en Unidad de Cuidados Paliativos del Hospital de Guadalajara. Ana Martín García es Psicóloga Clínica. La correspondencia sobre este artículo debe enviarse al primer autor al e-mail: srevengam@ sescam.jccm.es 
En la actualidad, en nuestro país, la formación en psicoterapia ofrece múltiples posibilidades. Por un lado se encuentra el itinerario formal del grado universitario y la residencia posterior para la obtención del título de Especialista en Psicología Clínica. Si bien la residencia incluye formación en más competencias que la psicoterapia, ésta es esencial durante la misma. Por otro lado, existen infinidad de máster, cursos, jornadas, talleres, etc, dónde también se persigue el aprendizaje de la psicoterapia en sus diversas formas. El pensar que estas formaciones son adecuadas y eficaces a la hora de convertirnos en mejores profesionales es algo que, como ya advierten Alberts y Edelstein (1990), tiende a darse por supuesto más que puesto a prueba. Sin embargo desde hace años empiezan a surgir corrientes investigadoras que tratan de abordar este asunto, por lo que procedemos a revisar brevemente algunos de estos trabajos.

Ford (1979), en una de las primeras revisiones sobre la eficacia de la formación, expone que, aunque hay resultados prometedores también existen limitaciones metodológicas que deben superarse. Más recientemente O'Donovan, Bain y Dyck (2005) comparan la eficacia de terapeutas que cursan un programa de postgraduado con aquellos que no lo cursan de tal forma que encuentran mejores resultados en el primer grupo, aunque las diferencias son sólo modestas. Por otro lado varios autores encuentran que la acumulación de años de entrenamiento no se traduce en una mejoría en cuanto a resultados clínicos. (Boswell, Castonguay y Wasserman, 2010; Erekson, Janis, Bailey, Cattani y Pedersen, 2017). En esta línea de resultados Malouff (2012), en una revisión de evidencia sobre los programas formativos estándares, pone en duda la contribución de éstos a los resultados terapéuticos positivos con pacientes.

Es interesante el trabajo de Owen, Wampold, Kopta, Rousmaniere y Miller (2016) donde observan un efecto positivo pero pequeño del entrenamiento a través de prácticums, programas predoctorales y postdoctorales. En este caso tratan de profundizar más en las causas de este efecto y encuentran que el crecimiento en los resultados es mayor cuando los pacientes son más leves pero tiende a desaparecer con los pacientes más graves. Por otro lado encuentran diferencias individuales en el crecimiento obtenido por los distintos terapeutas por lo que concluyen que no hay un efecto universal de la formación. Como conclusión final sugieren la necesidad de más investigaciones que vayan más allá de la medición cuantitativa de los resultados complementándose con aproximaciones cualitativas que aborden también el por qué de estas diferencias individuales.

La heterogeneidad de los estudios y la metodología empleada dificulta establecer conclusiones definitivas respecto a la eficacia de la formación. También resulta necesario aclarar que los resultados de estas investigaciones provienen de países con sistemas formativos que difieren del nuestro tanto en estructura como en contenido curricular por lo que no son directamente extrapolables al nuestro. Esto genera una cierta incertidumbre a la hora de enfrentar la pregunta de si nuestras vías de formación son adecuadas. Si bien estamos de acuerdo con la importancia de la acreditación y la certificación del itinerario curricular formal - ya que, como reconocen Hill, Spiegel, Hoffman, Kivlighan y Gelso (2017), al menos garantizan un mínimo de conocimiento y pericia y son coherentes con la ética profesional - también consideramos que los programas deben ser eficaces y por tanto traducirse en mejores resultados en la práctica clínica. Por ello retomamos la pregunta ya hecha por Binder (1993) hace años sobre si ha llegado el momento de mejorar la formación en psicoterapia. En este sentido nos parece especialmente apropiado acudir a los trabajos sobre la pericia y la búsqueda de la excelencia en psicoterapia (Prado-Abril, Sánchez-Reales e Inchausti, 2017) dado que pueden ofrecer orientaciones para mejorar la formación actual. Por ello procedemos a revisar brevemente estos planteamientos para posteriormente valorar su potencial utilidad. De aquí en adelante utilizaremos el término Pericia como equivalente a los conceptos de Excelencia o Expertise que se han utilizado también en la literatura.

\section{Pericia en Psicología Clínica}

La búsqueda de los mejores resultados en psicoterapia se ha focalizado en los tratamientos basados en evidencia. Prueba de ello es la gran cantidad de literatura existente y sus recapitulaciones en listas de tratamientos asociados a poblaciones diagnósticas (Chambless, 1996; Chambless y Hollon, 1998; Task Force on Promotion and Dissemination of Psychological Procedures, 1995). Esta aproximación ha demostrado su utilidad y para los profesionales en formación se convierte en un requisito el conocer y dominar estos trata- 
mientos. Pese a la utilidad de este acercamiento no deben confundirse los Tratamientos Basados en Evidencia con la Práctica Basada en Evidencia, definida por la APA (2006) como la combinación de dichos tratamientos con la pericia clínica de la persona que los aplica adaptándolos a las características, cultura y preferencias del paciente. En esta línea surgen las corrientes que plantean recuperar la importancia del terapeuta como parte implicada en los resultados de la psicoterapia.

Miller, Hubble, Chow y Seidel (2013) revisan la cuestión sobre los resultados de la psicoterapia y encuentran que el porcentaje de varianza atribuible al terapeuta se sitúa entre el 5 y el $9 \%$. Las diferencias en eficacia encontradas entre terapeutas al comparar unos con otros es un efecto constante encontrado a lo largo de la literatura (Baldwin e Imel, 2013; Miller, Hubble y Duncan, 2007; Wampold y Brown, 2005). Cuando se relacionan estas diferencias con los resultados obtenidos en contextos naturales se encuentra que contribuyen de forma pequeña pero significativa a los resultados, siendo el efecto superior a medida que aumenta el tiempo de psicoterapia (Goldberg, Hoyt, Nissen-lie, Nielsen y Wampold, 2016). Previo a las investigaciones de estos autores ya Okiishi, Lambert, Nielsen y Ogles (2003) encontraron que los denominados superpsicoterapeutas (supershrinks) - aquellos cuyos pacientes obtienen los mejores resultados - logran con sus pacientes una media de cambio 10 veces superior a la media de la muestra. Ante estos resultados resurge el interés por recuperar el foco en el terapeuta y esto lleva a algunos autores a retomar el concepto de Pericia en psicoterapia utilizado para ese segmento de terapeutas que alcanzan los niveles más altos de rendimiento (Hill et al., 2017; Norcross y Karpiak, 2017; Prado-Abril et al., 2017).

Hill et al. (2017) se enfrentan a la gran tarea de llegar a una definición de la Pericia y terminan por articularla como "la manifestación de los niveles más altos de capacidad, habilidad, competencia profesional y eficacia en psicoterapia”. Además de esto enriquecen la definición con criterios identificativos específicos como el desempeño, el funcionamiento cognitivo del clínico, los resultados obtenidos con del paciente, la experiencia, los aspectos personales y relacionales del profesional, las credenciales y el autocuestionamiento. Si bien el análisis exhaustivo del dilema de la definición excede el propósito de este trabajo remitimos al lector consultar los trabajos de Hill et al. (2017), Norcross y Karpiak (2017), Goodyear, Wampold, Tracey y Lichtenberget (2017) y en nuestro idioma Prado-Abril et al. (2017).

Existen dos aspectos del enfoque basado en la Pericia que nos interesa desarrollar debido a sus implicaciones en nuestra formación actual. Por un lado, los mecanismos a través de los cuales los clínicos con mayor Pericia llegan a serlo, puesto que potencialmente podemos utilizarlos como vías de formación. Por otro lado, la inclusión de los aspectos relacionales en la formación, los cuales ya son tenidos en cuenta por Hill et al. (2017) en su definición y que en las vías formativas actuales quedan generalmente relegados a un segundo plano.

\section{Mecanismos para desarrollar la Pericia}

Una de las cuestiones que se desprenden de los hallazgos del enfoque basado en la Pericia es cómo llegan a estos resultados los terapeutas con alta Pericia. Es fácil hipotetizar que la acumulación de experiencia debe ser la principal variable responsable de estos resultados, anticipando que aquellos psicoterapeutas más experimentados deben ser los que obtienen mejores resultados en la psicoterapia. No obstante, los estudios reflejan una realidad distinta. Stein y Lambert (1995) en su metaanálisis concluyen que la relación entre una mayor experiencia y la obtención de mejores resultados y menor tasa de abandono es modesta. Más recientemente, los resultados de Goldberg, Rousmaniere et al. (2016) muestran que la experiencia se relaciona de forma estadísticamente significativa pero pequeña con los resultados. Más allá de este dato lo que encuentran estos autores es que las diferencias entre unos terapeutas y otros son muy heterogéneas de tal forma que hay terapeutas que aprovechan mejor la experiencia consiguiendo un rendimiento más alto mientras que otros no sólo no la aprovechan si no que empeoran.

La escasa satisfacción encontrada en la acumulación de experiencia por sí misma como variable explicativa del rendimiento de los terapeutas que obtienen los mejores resultados lleva a la necesidad de ampliar la búsqueda y en esta línea Hill et al. (2017) postulan cinco mecanismos para llegar a la excelencia en la práctica 
clínica: la práctica deliberada, la supervisión, el feedback, la experiencia con clientes, y la terapia personal. A continuación procedemos a desarrollarlos brevemente.

El método identificado por diversos autores de forma consensuada como el más importante a la hora de aproximarse a la Pericia en psicoterapia es la práctica deliberada (Hill et al., 2017; Norcross y Karpiak, 2017; Goodyear et al., 2017), motivo por el cual centramos gran parte de nuestras reflexiones en este tipo de práctica.

La práctica deliberada se define como "el entrenamiento individualizado, diseñado especialmente por un entrenador o profesor para mejorar aspectos específicos de la ejecución individual, a través de la repetición y el perfeccionamiento sucesivo" (Lehmann, Ericcson y Anders, 1997). Si bien en su origen se aplica a otros campos diferentes de la Psicología Clínica, algunos autores han exportado el concepto a éste dada su utilidad. De esta manera el estudio de Chow et al. (2015) encuentra una relación positiva entre el tiempo que dedican los terapeutas a hacer práctica deliberada y los resultados obtenidos. También observan que los terapeutas que pasan más tiempo tratando de mejorar habilidades terapéuticas mediante actividades propias de la práctica deliberada - como revisar vídeos de sus propias actuaciones - obtienen mejores resultados con pacientes. Por otro lado, cuando la práctica clínica se hace en un contexto de práctica deliberada y feedback los terapeutas mejoran su rendimiento año a año (Goldberg et al., 2016).

Si se separan los componentes específicos que la conforman se encuentran cuatro componentes: esfuerzo focalizado y sistemático para mejorar la ejecución llevado a cabo durante un período prolongado, implicación y guía de un coach, mentor o profesor, feedback inmediato y continuo sobre habilidades individuales importantes y perfeccionamiento sucesivo y repetición a través de la práctica individual de este trabajo. Actualmente existe literatura dirigida a profesionales para que pongan en marcha las distintas estrategias de la práctica deliberada en su práctica clínica habitual (Rousmaniere, Goodyear, Miller y Wampold, 2017; Rousmaniere, 2016).

Junto a la práctica deliberada se ha propuesto la supervisión como otra herramienta que conduce a la Pericia. Los objetivos fundamentales de la supervisión, según la British Psychological Society, son proporcionar un espacio de consulta sobre los pacientes tratados, potenciar la calidad y competencia en la práctica, aportar un espacio que permita asumir desafíos de aprendizaje a la vez que permite el autocuidado y fomentar la continuidad del desarrollo profesional tanto del supervisado como del supervisor (2017). Los estudios muestran que se relaciona de forma moderada con los resultados en psicoterapia contribuyendo en un $16 \%$ de su varianza cuando se controlan los atributos del terapeuta y la gravedad inicial del paciente (Callahan, Almstrom, Swift, Borja y Heath, 2009). En una revisión posterior Watkins (2011) concluye que no se encuentra relación entre la supervisión y los resultados obtenidos en psicoterapia. Sin embargo también expone las limitaciones de los estudios realizados hasta la fecha de la revisión resaltando la necesidad de trabajos que ofrezcan más información. En estudios recientes se encuentra que la supervisión contribuye en menos del 1\% a los resultados sin embargo se reconoce la complejidad del estudio de este fenómeno por las múltiples variables mediadoras potencialmente influyentes (Rousmaniere, Swift, Babins-Wagner, Whipple y Berzins, 2016).

Algunos autores tratan de afinar más en esta cuestión estudiando la denominada como alianza de supervisión y las mejoras obtenidas tanto en satisfacción del supervisado como en su actuación en la clínica (Nor Mazlina, Wan Marzuki, Rohani y Sidek, 2016). De esta manera las supervisiones en las que se logra una alianza entre supervisor y supervisado permiten obtener mejores resultados de supervisión, esto es, mejor ejecución y satisfacción del supervisado. Por otro lado, algunos estudios hablan sobre los efectos de la supervisión inadecuada e incluso dañina (Ellis, Berger, Hanus, Ayala, Swords y Siembor, 2014; Ellis, 2010). Por tanto, es importante no considerar todas las supervisiones como equiparables e igualmente efectivas. En esta línea el meta-síntesis de Wilson, Davies y Weatherhead (2016) concluye que la supervisión debe abordar cuatro cuestiones centrales: su concepción como oportunidades de aprendizaje, la relación de supervisión, el poder en supervisión y el impacto de la supervisión. Atender a estos aspectos clave es esencial para que las supervisiones sean útiles para los supervisados y sus pacientes.

El Feedback es otro de los mecanismos más estudiados en los últimos años. Tal y como se entiende desde la psicología general, tiene una doble función: por un lado permite obtener información sobre un comportamiento; por otro, posibilita realizar cambios en la manifestación futura de dicho comportamiento (Claiborn y Goodyear, 2004). Tener más información, en forma de feedback, sobre cómo está funcionando o no una psicoterapia 
es especialmente útil dadas las tendencias de los clínicos a sobrevalorar los cambios positivos e infravalorar los negativos (Walfish, McAlister, O’Donnell y Lambert, 2012). De esta manera el clínico puede ajustar de forma más eficaz su comportamiento durante la terapia.

Además de obtener feedback de pacientes, se puede obtener de otras fuentes como supervisores u otros compañeros, especialmente cuando se cuentan con formatos audiovisuales o de supervisión en vivo. En los últimos años la investigación encuentra que la recapitulación sistemática del feedback que da el paciente al terapeuta se relaciona con resultados positivos (Gimeno-Peón, Barrio-Nespereira y Prado-Abril, 2018; Lambert y Shimokawa, 2011). En un estudio reciente se compara el tratamiento habitual sin la monitorización rutinaria de resultados con el tratamiento aplicando este tipo de feedback y se encuentra que este último grupo obtiene mejores resultados en la clínica (Brattland et al., 2018). Por otro lado la revisión realizada por Norcross y Lambert (2018) reconoce el empleo del feedback como un elemento de eficacia demostrada dentro de las relaciones empíricamente validadas.

En lo que se refiere a la práctica directa con pacientes encontramos que es uno de los mecanismos que, junto con la terapia personal, es percibido por los terapeutas como uno de los elementos más importantes en su desarrollo profesional (Hill y Knox, 2013). A la hora de profundizar en el efecto de la práctica directa con pacientes es interesante recordar los estudios mencionados anteriormente sobre el efecto de la acumulación de experiencia en los resultados clínicos obtenidos. Pese a su cuestionamiento los estudios encuentran un efecto pequeño o modesto sobre los resultados (Goldberg et al., 2016; Stein y Lambert, 1995).

El último mecanismo postulado para desarrollar la Pericia es la terapia personal. En la revisión de Macran y Shapiro (1998) concluyen que, dada la escasa investigación al respecto, no existen datos empíricos que avalen la relación entre recibir terapia personal y los resultados obtenidos en la práctica clínica sin embargo sí que se observan mejorías en variables del terapeuta relacionadas con los resultados, como la empatía o la autenticidad. A nivel teórico existen razones para pensar que puede tener un impacto en los resultados por su potencial efecto sobre el terapeuta en su empatía, autoconciencia, autoinsight, resolución de problemas personales y reconocimiento de la transferencia y contratransferencia (Hill et al., 2017).

\section{El papel de los aspectos relacionales en la búsqueda de la Pericia}

Nos parece pertinente que, hablando del desarrollo de la Pericia, nos detengamos brevemente en la contribución de los aspectos relacionales a este campo dado que quedaron relegados a un segundo plano tras la primera generación de compilaciones de tratamientos basados en la evidencia. La literatura al respecto concluye de forma robusta la necesidad de rescatar la figura del psicoterapeuta como profesional que no sólo es válido por su dominio de la técnica, sino también de las relaciones. En tanto en cuanto presente habilidades para el establecimiento de una sólida alianza terapéutica, adaptará adecuadamente las intervenciones al paciente y el contexto (Norcross y Lambert, 2018). Más concretamente, hay datos que apoyan que la alianza terapéutica constituye una competencia transversal a desarrollar en el psicoterapeuta en formación, tan responsable de los resultados del tratamiento como el método de intervención específico (Horvath, Del Re, Flückiger y Symonds, 2011). En el contexto de estos hallazgos, la APA Division of Psychotherapy Task Force ha emprendido la búsqueda de relaciones (terapéuticas) basadas en la evidencia - en lugar de tratamientos - con la finalidad de identificar los elementos eficaces de la relación terapéutica y métodos eficaces de adaptación de la terapia a la individualidad de los pacientes. En esta dirección, algunos autores han tratado de definir esta variable, entendiéndola como expertise relacional y describiéndola como la habilidad para establecer, mantener y emplear de forma creativa una relación de trabajo positiva con los pacientes (Skovnolt, Rennestad, Jennings, 1997).

Para el desarrollo de la Pericia encontramos como factor esencial el dedicar tiempo y formación al cuidado de los aspectos relacionales de la clínica así como cultivar las vías reconocidas por Hill et al. (2017) que conducen a la excelencia, especialmente la práctica deliberada, la supervisión y el feedback. Partiendo de estos desarrollos, reflexionamos en qué medida están integrados en nuestra formación como Psicólogos Clínicos durante la formación de la residencia y las implicaciones que ello supone para los profesionales, los residentes y la institución. 


\section{Los mecanismos para desarrollar la Pericia y el PIR}

Resulta necesario aclarar que este apartado está dotado de un carácter más subjetivo, puesto que está basado en nuestra propia experiencia como residentes. Asumimos que nuestra experiencia puede no ser universal y compartida por todos los residentes, sin embargo creemos que muchos compañeros y compañeras se identificarán con algunas reflexiones y sensaciones que planteamos en este trabajo.

Como punto de partida debemos hacer una breve introducción al proceso a través del cual se obtiene la especialización clínica en Psicología en España. La Formación Sanitaria Especializada de Psicólogos Clínicos, oficialmente regulada mediante el real decreto 2490/1998 del 20 de Noviembre, consta en la actualidad de cuatro años y destaca la importancia de la práctica profesional complementada con formación teórica. El sistema básico para la adquisición de las competencias necesarias es el de rotaciones en los diferentes dispositivos de Salud Mental donde los Psicólogos Internos Residentes progresan en diferentes niveles de autonomía y responsabilidad en un proceso tutorizado. Este sistema permite el aprendizaje con múltiples profesionales de diversas orientaciones al mismo tiempo que permite la adquisición de habilidades transversales que se desarrollan a lo largo de la residencia. De una forma general se establecen los objetivos, el circuito de rotaciones, las actividades y la figura de los tutores.

Para reflexionar en qué medida se cuenta en la formación PIR con los mecanismos propuestos por el enfoque basado en la Pericia, podemos empezar por acudir al Libro del Residente (Orden SAS/1620/2009), guía donde aparecen definidos las vías a través de los cuales los residentes van a adquirir las competencias propuestas. En este análisis, consideramos conveniente contrastar las bases de la formación planteadas en el Libro del Residente con nuestra propia experiencia formativa, donde en ocasiones nos alejamos del planteamiento inicial de esta guía. Concretamente, hemos focalizado en mayor medida en la reflexión acerca del lugar que ocupan la supervisión, el feedback y la práctica deliberada en nuestro proceso formativo, por ser los tres mecanismos que han demostrado mejores resultados. También consideramos que los tres están estrechamente relacionados entre sí y resulta interesante analizarlos de cara a la propuesta de cambios en la formación que nos aproximen a la pericia clínica. También dedicaremos parte de este apartado a la formación dentro del desempeño relacional.

Respecto a la supervisión, esta es definida en el Libro del Residente como uno de los pilares centrales de la formación, planteándose la necesidad de que esta sea reglada, focalizada en objetivos y adaptable a diferentes formatos (individual o grupal, directa o indirecta). El documento no entra a definir de una manera más sistemática el formato o las características de ésta, por lo que su delimitación queda en manos del supervisor y en todo caso del acuerdo que puedan alcanzar supervisor y supervisando. En la práctica diaria en ocasiones nos encontramos con que esta ausencia de regulación explícita de los espacios de supervisión la convierte en ocasiones en una actividad no del todo garantizada, tendiendo a variar notablemente entre unos hospitales y otros, y entre unos dispositivos y otros dentro del mismo hospital. Las consecuencias de esto se traducen en una supervisión que depende en mayor medida de variables relacionadas con la actitud e implicación de los residentes y facultativos de referencia. En este sentido, no podemos evitar poner en relación la falta de disponibilidad de espacios de supervisión con la sobrecarga asistencial bajo la cual se desempeña la profesión en la institución. En muchas ocasiones esto favorece que los profesionales con los que se va rotando durante la residencia no dispongan de espacios destinados a labores docentes con los residentes que se entienden como obligatorias en la propuesta formativa.

Como hemos podido observar en estudios previos, la calidad de la supervisión es una variable que influye notablemente. Sin embargo, encontramos que ésta no se evalúa de forma precisa y práctica, (si es que se evalúa), por parte de residentes y facultativos, quedando desatendidas variables que se relacionan con su buen rendimiento, como la alianza entre supervisando y supervisor (Nor Mazlina et al., 2016). Pensamos que tiene que ver por un lado con el desconocimiento de esta relación pero por otro por el temor a sentirse evaluados tanto supervisor como supervisado y a tener que gestionar una relación de supervisión que no está dando los resultados que deberían esperarse.

En relación con el mecanismo de feedback, podemos partir de que la propia relación con un supervisor implica recibir feedback ya sea a través de observaciones sobre el relato del residente u observaciones del propio trabajo en vivo del residente en sesión. Además, el feedback durante la residencia también se da de forma 
natural con los pacientes en la medida en que se contacta con ellos. El residente en mayor o menor medida se fija en el efecto que producen sus intervenciones en el paciente además de recoger el feedback que éste da. Sin embargo, no se hace referencia en ningún programa a su recogida sistemática. Consideramos que tiene que ver, como ocurría con la evaluación de la calidad de la supervisión, con el desconocimiento de su utilidad clínica pero también postulamos que exige por parte del residente un enfrentamiento a sus propios resultados arriesgándose a la recepción de críticas negativas, lo que puede dificultar su aplicación si no se cuenta con el apoyo necesario.

En relación al formato de práctica deliberada, algunas propuestas del programa formativo nos recuerdan a alguna de sus características. En esta línea el Libro del Residente hace algunas referencias a la individualización del programa dado que recoge la posibilidad de trabajar con objetivos personales acordados entre residente y tutor. Sin embargo la práctica deliberada es precisamente en esencia un entrenamiento individualizado de tal forma que no queda sólo en una sugerencia dentro de un cómputo de objetivos generales comunes a toda persona en formación. Otra diferencia la encontramos en la propia formulación de los objetivos dado que en el Libro del Residente se definen de forma general. Algunos ejemplos que encontramos son "Adquirir conocimientos sobre el fenómeno de las adicciones y los principales patrones de conductas adictivas" o "Adquirir y aplicar conocimientos sobre los principales programas de psicoterapia y procedimientos de intervención y tratamiento psicológicos empíricamente validados en adicciones". Entendemos que "partiendo desde la práctica deliberada" los objetivos deberían ser más específicos en lo que a habilidades y aprendizajes se refiere, tomando como referencia la línea base del desempeño del residente y proporcionando feedback específico e inmediato sobre habilidades y competencias a entrenar. Observamos también, que la cantidad de actividad asistencial que se realiza adquiere un peso significativo a la hora de valorar el cumplimiento de objetivos del residente puesto que se sugieren en gran parte de los objetivos frecuencias necesarias para considerarlo como superado. En este sentido consideramos, en la línea del enfoque basado en la Pericia, que la mera acumulación de experiencia en una determinada práctica no conduce a su dominio. De esta manera un enfoque más coherente con la práctica deliberada debería centrarse más en la calidad de la práctica en cuanto a adquisición de habilidades se refiere y no tanto en la cantidad de veces que se realiza.

Por otro lado, existe un problema para dar continuidad a la adquisición de habilidades de forma individualizada. Cada residente requiere tiempos distintos para consolidar los aprendizajes exigidos por el programa sin embargo, la duración de cada rotación está definida de antemano haciendo difícil que un residente que no ha adquirido aún una determinada habilidad pueda continuar con su entrenamiento. Si bien consideramos, que es necesario establecer un programa fijo con tiempos establecidos dada la importancia de la acreditación consideramos que esto en ocasiones dificulta el seguir la filosofía de la individualización de la formación de la Práctica Deliberada.

Otra cuestión que se añade es la del feedback inmediato. En el programa no se establecen de forma explícita espacios que permitan al residente recibir un feedback lo suficientemente próximo en el tiempo para considerarlo inmediato. A menudo las supervisiones de casos se hacen pasados varios días o incluso semanas. Desde la Práctica Deliberada se solventa esta situación a través de mecanismos como la grabación en vídeo de las sesiones y su supervisión a posteriori de tal forma que aunque el feedback sea espaciado en el tiempo es específico y dirigido a componentes concretos de la actuación del aprendiz (Prado-Abril, Gimeno-Peón, Inchausti, Sánchez-Reales, 2019). En este caso nuestra experiencia revela que no es una práctica habitual durante la residencia, si bien es posible que algunos hospitales lo hagan.

Con respecto a la formación en aspectos relacionales del desempeño clínico, nos encontramos con el mensaje constante de la importancia de formarse y adquirir pericia en estos aspectos, no obstante en la práctica no se le otorga un lugar definido a esta área. Por este motivo nos resulta relevante visibilizar el contraste entre la escasez de formación explícita recibida acerca de los aspectos relacionales de la psicoterapia y la relevancia que se le otorga en la investigación. Tal vez esto ocurre por darse por adquiridas estas habilidades o tal vez por focalizarse excesivamente en los aspectos técnicos de la psicoterapia.

Podemos concluir que el programa PIR cuenta con elementos próximos a los mecanismos postulados por el enfoque basado en la Pericia para el desarrollo de la excelencia pero aún guarda cierta distancia con su aplicación óptima en base a estos criterios. Contamos principalmente con práctica con supervisión y feedback 
(aunque éste no sea sistematizado), pero estas actividades formativas no adquieren el formato planteado desde la Práctica Deliberada, aun siendo enmarcada como la vía más relevante para el desarrollo de la Pericia (Hill et al., 2017).

Tras analizar la realidad del residente, planteamos propuestas para acercarnos a la Pericia en la clínica.

\section{¿Cómo aproximarnos al enfoque basado en la Pericia? Algunas reflexiones}

Nuestra formación actual reúne condiciones que, en contraste con otras propuestas formativas menos exhaustivas (cursos de experto, másteres...), favorece la revisión y perfeccionamiento sucesivo de la práctica clínica en una amplia diversidad de contextos y durante un período de tiempo prolongado, así como la disponibilidad de figuras profesionales especializadas que supervisen el desempeño en la práctica clínica. No obstante, sentimos la necesidad de plantearnos potenciales cambios que permitan que los residentes iniciemos el camino hacia la excelencia profesional ya desde esta etapa inicial de nuestra formación. Más que dar con medidas específicas, pretendemos hacer propuestas generales que sirvan como estímulo para el debate y la reflexión persiguiendo de esta manera la integración de nuevas maneras de aprender en la formación PIR que nos permitan acercarnos a la pericia clínica.

\section{Difusión de la práctica basada en evidencia y del enfoque basado en la Pericia}

En primer lugar, consideramos necesario rescatar la figura del profesional dentro de la Psicología Clínica. En la era de los tratamientos basados en evidencia se difumina la importancia del clínico que los aplica y su contribución a los resultados. Por este motivo creemos esencial dar a conocer estos planteamientos que complementan la formación en tratamientos. A su vez, conocer los mecanismos para el desarrollo de la Pericia es el primer paso necesario para poder aplicarlos a la formación.

\section{Desarrollo de programas de evaluación e investigación que permitan avalar y/o mejorar los programas} formativos actuales

Pensamos que no se debe dar por sentada la eficacia de nuestro programa formativo y es necesaria su evaluación. Pese a que entendemos la dificultad de enfrentarse a tal tarea, las líneas de investigación dirigidas a este objetivo pueden acercarnos a lo que entenderíamos como un programa formativo basado en evidencia. En este sentido queremos destacar que existen trabajos en nuestro entorno que, aunque no están dedicados a evaluar los programas formativos de nuestro país, sí que investigan sobre la Pericia y la Práctica Deliberada (Prado-Abril et al., 2019), el Feedback y la Monitorización Sistemática de Resultados (Gimeno-Peón et al., 2018) y los procesos relacionales y de alianza en psicoterapia (Andrade-González, Lahera y Fernández-Liria, 2017).

\section{Mejora de la calidad de la supervisión y facilitar el feedback inmediato}

Todos los elementos que permitan la supervisión y el posterior feedback del supervisor son susceptibles de aproximarnos a la práctica deliberada y por tanto a la Pericia. Dado el lugar protagonista que consideramos que tiene la supervisión en la formación del Psicólogo Clínico, consideramos que ésta debe ser de la mejor calidad posible. Por eso creemos que debe someterse a la evaluación de su calidad con el fin de mejorarla. No todas las supervisiones son iguales y en ese sentido el cómo se hace la supervisión tiene una gran importancia (p.e. Ellis et al., 2014; Ellis, 2010; Nor Mazlina et al., 2016).

Respecto al formato, existen medios que facilitan la supervisión, tales como el empleo de espejos unidireccionales o la grabación en vídeo de las sesiones. En esta línea se han hallado resultados positivos con métodos 
de supervisión en vivo, como el formato bug-in-the-eye (Probst, Jakob, Kaufmann, Müller-Neng, Bohus y Weck, 2018). El feedback de los pacientes podría ser recogido a través de escalas de escasa elaboración que resulten ágiles y no pesarosas para los pacientes, con la finalidad de que esta información también contribuya al progresivo aprendizaje y perfeccionamiento de habilidades terapéuticas.

\section{Individualización de los objetivos formativos}

Aproximarnos a la práctica deliberada implica la individualización del feedback y del desarrollo formativo. Es una realidad el hecho de que llegamos a la residencia desde distintos itinerarios formativos y con distinto nivel de habilidad en cada uno de los dominios requeridos para la psicoterapia. Por tanto, no partimos todos de un mismo lugar ni nos planteamos las mismas metas. Incluir espacios formativos individualizados basados en la práctica deliberada podría complementar - sin necesidad de sustituir - nuestra formación actual.

También en la línea de individualizar, sería interesante la regulación explícita de la coordinación entre los supervisores de los diferentes dispositivos, ya que el cambio de supervisión de un adjunto a otro cuando rotamos de dispositivo dificulta la continuidad del entrenamiento en los objetivos individualizados por parte del residente. Si bien también ponemos en cuestión el realismo que tiene este planteamiento, por la notable inversión de tiempo añadida que supondría al trabajo de los facultativos.

\section{Fomento del aprendizaje activo y experiencial}

Nos resulta fundamental la inclusión de métodos formativos menos pasivos, más aproximados a la práctica deliberada. Un ejemplo de la potencialidad de estos espacios serían las sesiones clínicas llevadas a cabo por los residentes durante la formación. Son entendidas como lugares donde exponer casos complejos y favorecer el debate y la reflexión acerca de los mismos. No obstante, partiendo de nuestra propia experiencia, observamos en estos espacios el riesgo de que adquieran un formato expositivo, donde los residentes traen casos cerrados que han sido abordados por sus adjuntos con construcciones teóricas e intervenciones ya cerradas. Esto deja poco margen para la exposición de lo "bueno" y lo "malo", aspecto muy relacionado con la cultura de hermetismo con los errores que existe en la institución . La oportunidad de que todos, residentes y especialistas, podamos compartir las propias dificultades convierte esos espacios en lugares seguros donde entendernos como personas con sanas vulnerabilidades en proceso de aprendizaje continuo. Este clima favorece en mayor medida la apertura a revisar y perfeccionar la práctica clínica de forma constante.

En relación con esta propuesta, nos resulta de especial importancia hacer mención a un espacio concreto de docencia establecido en la formación de los residentes de Salud Mental del Complejo Hospitalario de Toledo, denominado "Grupo de Estudio", del cual han partido gran parte de las reflexiones de este trabajo y que creemos refleja en cierta medida las potencialidades del aprendizaje activo al que hacemos referencia. El encuadre del espacio es descrito de forma más detallada en el próximo apartado. Nuestra propia experiencia en espacios como éste nos reafirma en la necesidad de diseñar formatos de docencia que faciliten aprendizajes experienciales y activos, donde el material pueda ser elaborado, reflexionado, cuestionado, etc. y por tanto, integrado desde un lugar más individualizado.

\section{Facilitación de espacios y tiempos a los adjuntos para la formación}

La mayor parte de las propuestas formativas planteadas requieren la necesidad de que la figura del facultativo tenga disponibilidad para poder realizar labores de supervisión con los residentes. La presión asistencial es una variable que, a parte de limitar el tiempo físico del que dispone el facultativo, va a repercutir en la calidad de la supervisión en tanto en cuanto se convierte en un factor de estrés significativo para el adjunto, al que se le 
añade otra tarea profesional que acaba realizando de forma más superficial, en espacios informales no destinados a la supervisión, o directamente no la lleva a cabo.

\section{Abordaje explícito de competencias relacionales en la formación del clínico}

Un riesgo en el que podemos caer fácilmente es dar por adquirida la capacidad para establecer una alianza terapéutica exitosa en el profesional por el simple hecho de que conozca y domine los aspectos técnicos de la psicoterapia. Si entendemos la complejidad de la relación terapeuta-paciente y la multiplicidad de variables que influyen en la misma (relacionadas con el paciente, el profesional, el contexto de tratamiento, y la propia relación en sí), podemos valorar la necesidad de incluir contenidos o formatos de docencia que aborden y trabajen de forma explícita con las habilidades y competencias relacionales de los residentes.

\section{Discusión}

El origen de este trabajo proviene de un espacio de docencia del Complejo Hospitalario de Toledo que denominamos Grupo de Estudio. Se trata de un espacio constituido tanto por residentes (de Psicología Clínica, Enfermería, Psiquiatría y otras especialidades que se encuentren rotando en los dispositivos de salud mental del hospital) como por facultativos, donde se trabaja material en diferentes formatos con la finalidad de favorecer la reflexión crítica y la elaboración del contenido de forma grupal, existiendo una figura de coordinador que gestiona la administración del material y la dinámica grupal. Consideramos que se trata de un espacio donde se ponen en marcha procesos que facilitan en el residente la oportunidad de pensarse como profesional en un contexto donde todos los miembros pueden permitirse dudar y compartir inquietudes, primándose la actitud reflexiva y la disponibilidad para elaborar el material por encima de la adquisición de conocimientos de carácter acumulativo. Partiendo del trabajo de Prado-Abril et al. (2017) acerca de la pericia y excelencia en Psicología Clínica que revisamos en una de las sesiones del Grupo de Estudio, llevamos a cabo la puesta en común de las reflexiones planteadas previamente acerca de nuestro programa formativo actual y las implicaciones que se pueden derivar de asumir un enfoque basado en la Excelencia en nuestra propia formación como residentes, que describimos a continuación.

Una de las preguntas que más espacio y reflexión requirió fue ¿cuáles serían las implicaciones de integrar y sistematizar prácticas vinculadas al logro de la excelencia en la práctica clínica? Una forma de organizar nuestras reflexiones puede ser entender las implicaciones que existen para los profesionales (residentes y facultativos), los pacientes y la institución. Resulta imprescindible aclarar que los cambios en un nivel inevitablemente afectan a otros, por lo tanto las implicaciones que puede tener para los profesionales se interrelacionan tanto con la institución como con los pacientes que atienden, no obstante lo estructuramos en estos apartados para simplificar y hacer más sencilla la lectura de los mismos.

\section{Implicaciones para los residentes}

Una formación de residentes que adopte el formato de práctica deliberada incluye la presencia de un observador externo que ofrece un feedback constante acerca del desempeño individual del residente. Esto favorece una mayor conciencia acerca de la propia práctica clínica, lo que a la larga se traduce en una revisión y perfeccionamiento permanentes de la misma. Si desde el inicio de nuestra formación profesional incluimos esta variable, es esperable que disminuya la probabilidad de adoptar rutinas rígidas e inflexibles de intervención que no se adapten a los pacientes ni evolucionen con el tiempo además de aumentar la propia capacidad de autocrítica. Otra ventaja para los residentes, que se extiende también a los facultativos, consiste en mantener en mente de forma constante el objetivo último, que es propiciar al paciente el mejor cuidado posible. Este objetivo requiere la revisión de los errores profesionales para poder resolverlos. Cuando los errores se ocultan perdemos la meta de nuestra propia profesión, emprendiendo acciones que van más en la dirección de proteger nuestro propio ego que de cuidar al paciente. 
No obstante, también existen riesgos respecto a la inclusión de estos estilos formativos, que pueden favorecer en los residentes resistencias a adoptar este enfoque. Uno de los elementos intrínsecos a estas prácticas es la exposición a la que se ve sometido el residente. Las competencias, las habilidades terapéuticas, los conocimientos, etc. quedan expuestos ante la mirada crítica de un supervisor que sitúa las propias dificultades en el punto de mira de cara al perfeccionamiento de las mismas. Además, en formatos de supervisión grupal el temor se extiende a la evaluación de los compañeros. A esta circunstancia debemos añadirle el hecho de que aquellos que inician la residencia, generalmente en un proceso de adaptación a esta nueva profesión, lo hacen desde una condición de inexpertos, con la inseguridad que esto conlleva. Más intimidante todavía resulta el hecho de que las propias dificultades personales y aspectos relacionados con nuestra propia personalidad y psicobiografía también quedan expuestos ante uno mismo y el supervisor externo, ya que, inevitablemente, por las características de esta profesión, influyen y modulan el "Yo profesional". Enfrentarse a la supervisión, la recepción de feedback por parte de profesionales o pacientes o formatos de práctica deliberada implica mirar y reconocer estas dificultades para evitar que obstaculicen el desarrollo profesional. Todos estos aspectos suponen el reto al que los residentes deben enfrentarse cuando se adopta este tipo de formación.

Sin embargo es importante recordar los estudios ya comentados en espacios anteriores donde aparecen variables mediadoras que permiten afrontar estas dificultades como la alianza de supervisión (p. e. Nor Mazlina et al., 2016). La experiencia se vuelve más constructiva cuando se establece una relación supervisora de confianza. Si no se da esta cualidad de la relación es fácil que el supervisado caiga en el intento de ocultar sus dificultades además de los procesos contratransferenciales (Safran y Muran, 2005). Por tanto consideramos relevante para la cuestión promover no sólo mayor cantidad de supervisión si no también de mayor calidad.

\section{Implicaciones para los facultativos que supervisan y tutorizan}

La práctica deliberada también entraña potenciales beneficios para la figura del supervisor externo. Partimos de la base de que de forma frecuente $-\mathrm{y}$ teniendo en cuenta que existen excepciones - las condiciones de trabajo de los psicólogos clínicos en el sistema de salud público una vez obtienen la especialidad dejan de favorecer el aprendizaje desde la práctica deliberada. Los espacios de formación se ven reducidos a cursos donde prevalece el aprendizaje de tipo pasivo, la elevada presión asistencial dificulta el encuentro de espacios donde coordinarse con colegas y revisar la propia práctica clínica. En determinados dispositivos las limitaciones son incluso mayores, pudiendo verse en la circunstancia de permanecer solo en el despacho durante toda la jornada laboral atendiendo pacientes a un ritmo frenético que no favorece la reflexión.

La oportunidad de participar en formación desde la práctica deliberada con los residentes se convierte en una oportunidad de revisión y actualización del propio quehacer profesional del facultativo. Consideramos que formar a residentes también es una forma de evaluación continua y aumenta la conciencia sobre el sentido de las propias intervenciones. Las preguntas o dificultades que puedan plantear los residentes favorecen en los supervisores la conexión con el aprendizaje activo y el abandono de las pautas rígidas.

A la hora de valorar las dificultades a las que estos estilos formativos enfrentan a los supervisores podemos extrapolar similares reflexiones a las que hemos hecho con los residentes: el espacio se convierte en un lugar donde también se ponen a prueba los conocimientos y competencias del supervisor. En este sentido la posición de experto que asume el adjunto puede llevarle a entenderse a sí mismo como el portador del conocimiento que debe ser demostrado quedando libre de la duda, más propia y entendible en el residente. Esta situación confronta también al facultativo con sus propias dificultades e inseguridades.

\section{Implicaciones para los pacientes}

Para favorecer la revisión de la práctica profesional se debería contar con el feedback de los pacientes acerca de sus propia evolución y su visión de la labor del profesional. Esto implicaría otorgarles un papel más activo en su propio proceso terapéutico. Además, el hecho de invertir en una formación de mayor calidad a la larga debería 
traducirse en mejores resultados en los tratamiento psicológicos, que a largo plazo también supone un ahorro de recursos (disminución de prescripciones de psicofármacos, procesos psicoterapéuticos de mayor brevedad...).

\section{Implicaciones para la institución}

Existen claras barreras institucionales que dificultan la instauración de la práctica deliberada en la formación de residentes. Algunas de ellas han sido mencionadas con anterioridad (p. e. la elevada presión asistencial en las agendas de los facultativos). Además, desde nuestra propia experiencia percibimos que la filosofía formativa que impera en la actualidad en las instituciones sanitarias favorece el aprendizaje pasivo, llegando a castigar la presencia de miedos, dudas o inseguridades entre los residentes en formación en las diferentes especialidades. Entre los profesionales ya especialistas, los propios errores pueden ser vividos en mayor medida como una oportunidad de juicio, más que como una oportunidad de cambio, lo que a largo plazo se traduce en un hermetismo respecto a los propios fallos que construye profesionales "defendidos", con un método rígido de actuación que dificulta la apertura al cambio y la flexibilidad. Esta cultura es la "rueda" en la que los residentes tienen el riesgo de entrar durante sus años de formación.

La instauración de una formación próxima a la práctica deliberada debe venir de la mano de un cambio en la cultura institucional respecto a la idea del conocimiento finito en lo que respecta a la salud mental, a favor de una concepción del conocimiento y de la formación profesional de psicólogos clínicos como algo dinámico, en continua construcción, donde la incertidumbre es un ingrediente que siempre acompaña y la existencia de la verdad absoluta es cuestionable. Una forma de favorecer este pensamiento podría ser facilitar el aprendizaje de las diferentes orientaciones teóricas y aplicaciones técnicas en la práctica clínica con la apertura suficiente como para no asumir ninguna de ellas de forma dogmática, pudiendo entender la aplicabilidad de cada una en función del contexto y el paciente, fomentando así la flexibilidad. Otra dinámica que podría estimular este pensamiento es favorecer en contextos docentes (cursos, sesiones clínicas, seminarios...) un ambiente que no evalúe o enseñe desde un lugar pasivo, si no que favorezca que los residentes puedan generar sus propias respuestas y su propio estilo terapéutico de la mano del conocimiento que vayan adquiriendo.

\section{Conclusiones}

A nivel general, podemos extraer una conclusión basada en la evidencia encontrada hasta el momento sobre la formación y los distintos mecanismos que la conforman, y es que no podemos responder a preguntas tan generales cómo “¿funciona la formación?” “¿Es eficaz la supervisión?” o “¿Es igualmente eficaz o transferible la formación entre las distintas culturas?” Abordar estas cuestiones implica ir más allá elaborando preguntas que incluyan qué formación, con qué persona, en qué contexto relacional, etc. La complejidad del trabajo clínico se traduce inevitablemente en complejidad a la hora de entender la formación clínica de calidad.

Nos damos cuenta también de que la evaluación de nuestro propio desempeño y nuestra formación implica sembrar dudas que pueden amenazar nuestra autoestima profesional. Esto ya ocurrió en otros momentos del pasado cuando el famoso estudio de Eysenck cuestionaba la efectividad de los tratamientos psicológicos (Eysenck, 1952). Si bien fueron momentos difíciles para la profesión, poner en duda la eficacia de la psicoterapia impulsó el desarrollo de los tratamientos basados en evidencia. Poner en duda nuestra formación implica asumir el riesgo de colocarnos frente a una realidad incómoda, sin embargo confiamos en que también permitirá su potenciación. Más aún cuando la investigación sobre los mecanismos para llegar a la pericia profesional empieza a mostrar el camino a seguir.

No obstante, los avances en la investigación también entrañan un riesgo de sobre-sistematizar el aprendizaje de un proceso como la psicoterapia, que tiene mucho de creativo, flexible e impredecible. Resulta necesario poder encontrar un equilibrio entre la regulación de una formación de calidad que incluya elementos como el feedback, la supervisión y la práctica deliberada, y el mantenimiento de espacios abiertos de reflexión, donde exista la posibilidad de que surjan formas distintas de enfrentarse al trabajo clínico que precisamente darán 
salida a las dificultades que puedan surgir con pacientes desde un lugar más personalizado y adaptado a sus necesidades. Precisamente porque somos un sistema abierto de interrelaciones, es deseable respetar espacios de intercambio e influencia mutua no protocolizados.

Muy vinculado a esta necesidad está la de cuidar y respetar la alianza terapéutica, dotada de una complejidad y una potencia terapéutica mayor que la que en ocasiones le otorgamos, y que debemos entender que posee unas características especiales que deben ser atendidas y cuidadas durante todo el proceso terapéutico, y por tanto supervisadas. Inevitablemente nuestro propio proceso y nuestra forma de relacionarnos va a influir en nuestras habilidades relacionales a nivel profesional. Esto de nuevo sugiere lo conveniente de poder revisarnos y elevar la conciencia sobre nuestros propios conflictos y nuestro estilo relacional en tanto en cuanto éstos influyan en nuestra labor como clínicos.

\section{Referencias}

APA Presidential Task Force on Evidence-Based Practice. (2006). Evidence-based practice in psychology. American Psychologist, 61, 271-285. https://doi.org/10.1037/0003-066X.61.4.271

Alberts, G. y Edelstein, B. (1990). Therapist training: A critical review of skill training studies. Clinical Psychology Review 10, 497-511. https://doi.org/10.1016/0272-7358(90)90094-Q

Andrade-González, N., Lahera, G. y Fernández-Liria, A. (2017). Patient-Therapist Perspective of the Working Alliance in Psychotherapy. The Psychiatric quarterly, 88, 623-633. https://doi.org/10.1007/s11126-0169477-4

Baldwin, S. A. e Imel, Z. E. (2013). Therapist effects: Findings and methods. En M. J. Lambert (Ed.), Bergin and Garfield's handbook of psychotherapy and behaviorchange (6 ${ }^{\mathrm{a}}$ ed., pp. 258-297). Hoboken, NJ: Wiley.

Binder, J. L. (1993). Is it time to improve psychotherapy training? Clinical Psychology Review, 13, 301-318. https://doi.org/10.1016/0272-7358(93)90015-E

Boswell, J. F., Castonguay, L. G., y Wasserman, R. H. (2010). Effects of psychotherapy training and intervention use on session outcome. Journal of Consulting and Clinical Psychology, 78, 717-723. https://doi. org/10.1037/a0020088

Brattland, H., Koksvik, J. M., Burkeland, O., Gråwe, R. W., Klöckner, C., Linaker, O. M., ... Iversen, V. C. (2018). The effects of routine outcome monitoring (ROM) on therapy outcomes in the course of an implementation process: A randomized clinical trial. Journal of Counseling Psychology, 65, 641-652. http://doi. org/10.1037/cou0000286

British Psychological Society. (2017). Practice Guidelines. (Third Edition). Recuperado de https://www.bps. org.uk/news-and-policy/practice-guidelines

Division 12 Board. (1993). Task Force on Promotion and Dissemination of Psichological Procedures. Recuperado de: http://www.div12.org/sites/default/files/InitialReportOfTheChamblessTaskForce.pdf

Callahan, J. L., Almstrom, C. M., Swift, J. K., Borja, S. E. y Heath, C. J. (2009). Exploring the contribution of supervisors to intervention outcomes. Training and Education in Professional Psychology, 3(2), 72-77. https://doi.org/10.1037/a0014294

Chambless, D. L. (1996), In Defense of Dissemination of Empirically Supported Psychological Interventions. Clinical Psychology: Science and Practice, 3: 230-235. doi:10.1111/j.1468-2850.1996.tb00074.x

Chambless, D. L. y Hollon, S. D. (1998). Defining empirically supported therapies. Journal of Consulting and Clinical Psychology, 66, 7-18. http://dx.doi.org/10.1037//0022-006X.66.1.7

Chow, D. L., Miller, S. D., Seidel, J. A., Kane, R. T., Thornton, J. A. y Andrews, W. P. (2015). The role of deliberate practice in the development of highly effective psychotherapists. Psychotherapy, 52, 337-345. https:// doi.org/10.1037/pst0000015

Claiborn, C. D. y Goodyear, R. K. (2004). Feedback in psychotherapy. Journal of Clinical Psychology, 61, 209-217. https://doi.org/10.1002/jclp.20112

Ellis, M. V. (2010). Bridging the Science and Practice of Clinical Supervision: Some Discoveries, Some Misconceptions. The Clinical Supervisor, 29(1), 95-116. https://doi.org/10.1080/07325221003741910 
Ellis, M. V., Berger,L., Hanus, A. E., Ayala, E. E., Swords, B. A. y Siembor, M. (2014). Inadequate and Harmful Clinical Supervision. The Counseling Psychologist,42,434-472. https://doi.org/10.1177/0011000013508656

Erekson, D. M., Janis, R., Bailey, R. J., Cattani, K. y Pedersen, T. R. (2017). A longitudinal investigation of the impact of psychotherapist training: Does training improve client outcomes? Journal of Counseling Psychology, 64, 514-524. https://doi.org/10.1037/cou0000252

Eysenck, H. J. (1952). The effects of psychotherapy: an evaluation. Journal of Consulting Psychology, 16, 319-324. https://doi.org/10.1037/h0063633

Ford, J. D. (1979). Research on Training Counselors and Clinicians. Review of Educational Research, 49(1), 87-130. https://doi.org/10.3102/00346543049001087

Gimeno-Peón, A., Barrio-Nespereira, A. y Prado-Abril, J. (2018). Monitorización Sistemática y Feedback en Psicoterapia. Papeles del Psicólogo, 39, 174-182. https://doi.org/10.23923/pap.psicol2018.2872

Goldberg, S., Babins-Wagner, R., Rousmaniere, T., Berzins, S., Hoyt, W. T., Whipple, J., ... Wampold, B. E. (2016). Creating a Climate for Therapist Improvement: A Case Study of an Agency Focused on Outcomes and Deliberate Practice. Psychotherapy Theory Research Practice Training 53, 367-375. https://doi. org/10.1037/pst0000060

Goldberg, S. B., Hoyt, W. T., Nissen-Lie, H. A., Nielsen, S. L. y Wampold, B. E. (2016). Unpacking the therapist effect: Impact of treatment length differs for high- and low-performing therapists. Psychotherapy Research. https://doi.org/10.1080/10503307.2016.1216625

Goldberg, S., Rousmaniere, T. G., Miller, S. D., Whipple, J., Nielsen, S. L., Hoyt, W. T. y Wampold, B. E. (2016). Do psychotherapists improve with time and experience? A longitudinal analysis of outcomes in a clinical setting. Journal of Counseling Psychology, 63, 1-11.

Goodyear, R. K., Wampold, B. E., Tracey, T. J. G. y Lichtenberg, J. W. (2017). Psychotherapy Expertise Should Mean Superior Outcomes and Demonstrable Improvement Over Time. The Counseling Psychologist, 45(1), 54-65. https://doi.org/10.1177/0011000016652691

Hill, C. E., Spiegel, S. B, Hoffman, M. A., Kivlighan, D. M. y Gelso, C. J. (2017). Therapist expertise in psychotherapy revisited. The Counseling Psychologist, 45(1), 7-53. https://doi.org/10.1177/0011000016641192

Hill y Knox. (2013). Training and supervision in psychotherapy. En M. J. Lambert (Ed.), Bergin and Garfield's handbook of psychotherapy and behavior change (pp. 775-811). Hoboken, NJ: Wiley.

Horvath, A. O., Del Re, A. C., Flückiger, C. y Symonds, D. (2011). Alliance in Individual Psychotherapy. Psychotherapy, 48(1), 9-16. https://doi.org/10.1037/a0022186

Lambert, M. y Shimokawa, K. (2011). Collecting Client Feedback. Psychotherapy Theory Research Practice Training 48(1), 72-9. https://doi.org/10.1037/a0022238

Lehmann, A. C, Ericsson, K. A., Anders, K. (1997). Research on expert performance and deliberate practice: Implications for the education of amateur musicians and music students. Psychomusicology: A Journal of Research in Music Cognition, 16(1-2), 40-58. https://doi.org/10.1037/h0094068

Macran, S. y Shapiro, D. A. (1998). The role of personal therapy for therapists: A review. British Journal of Medical Psychology, 71(1), 13-25. https://doi.org/10.1111/j.2044-8341.1998.tb01364.x

Malouff, J. (2012). The need for empirically supported psychology training standards. Psychotherapy in Australia, 18(3), 28-32.

Miller, S. D., Hubble, M. A., Chow, D. L. y Seidel, J. A. (2013). The Outcome of Psychotherapy: Yesterday, Today, and Tomorrow. Psychotherapy, 50(1), 88-97. https://doi.org/10.1037/a0031097

Miller, S. D., Hubble, M. A. y Duncan, B. L. (2007). Supershrinks: Learning from thefield's most effective practitioners. Psychotherapy Networker, 31, 26-35

Nor Mazlina, G., Wan Marzuki W. J., Rohani, A. T. y Sidek M. N. (2016). Influence of Supervisees’ Working Alliance on Supervision Outcomes: A Study in Malaysia Context. International Journal of Social Science and Humanity, 6(1), 9-13. https://doi.org/10.7763/IJSSH.2016.V6.609

Norcross, J. C. y Karpiak, C. P. (2017). Our best selves: Defining and actualizingexpertise in psychotherapy. The Counseling Psychologist, 45, 66-75.

Norcross, J. C. y Lambert, M. J. (2018). Psychotherapy relationships that work III. Psychotherapy, 55, 303315. https://doi.org/10.1037/pst0000193 
O’Donovan, A., Bain, J. D. y Dyck, M. J. (2005). Does Clinical Psychology Education Enhance the Clinical Competence of Practitioners?. Professional Psychology Research and Practice. 36(1), 104-111. https://doi. org/10.1037/0735-7028.36.1.104

Okiishi, J. C., Lambert, M. J., Nielsen, L. y Ogles, B. M. (2003). Waiting for supershrink:An empirical analysis of therapist effects. Clinical Psychology \& Psychotherapy,10, 361-373. https://doi.org/10.1002/cpp.383

Owen, J., Wampold, B. E., Kopta, M., Rousmaniere, T. y Miller, S. D. (2016). As good as it gets? Therapy outcomes of trainees over time. Journal of Counseling Psychology, 63(1), 12-19. https://doi.org/10.1037/ cou0000112

Prado-Abril, J., Gimeno-Peón, A., Inchausti, F. y Sánchez-Reales, S. (2019). Pericia, efectos del terapeuta y práctica deliberada: el ciclo de la excelencia. Papeles del Psicólogo, artículo en prensa. https://doi. org/10.23923/pap.psicol2019.2888

Prado-Abril, J., Sánchez-Reales, S., e Inchausti, F. (2017). En busca de nuestra mejor versión: pericia y excelencia en Psicología Clínica. Ansiedad y Estrés, 23(2-3), 110-117. https://doi.org/10.1016/j.anyes.2017.06.001

Probst, T., Jakob, M., Kaufmann, Y. M., Müller-Neng, J. M. B., Bohus, M. y Weck, F. (2018). Patients' and therapists' experiences of general change mechanisms during bug-in-the-eye and delayed video-based supervised cognitive-behavioral therapy. A randomized controlled trial. Journal of Clinical Psychology, 74, 509-522. https://doi.org/10.1002/jclp.22519

Rousmaniere, T. (2016). Deliberate Practice for Psychotherapists. A Guide to Improving Clinical Effectiveness. Nueva York, Estados Unidos: Routledge.

Rousmaniere, T. G., Swift, J. K., Babins-Wagner, R., Whipple, J. L. y Berzins, S. (2016). Supervisor variance in psychotherapy outcome in routine practice. Psychotherapy Research, 26, 196-205. https://doi.org/10.108 0/10503307.2014.963730

Rousmaniere, T., Goodyear, R. K., Miller, S. D. y Wampold, B. E. (2017). The Cycle of Excellence: Using Deliberate Practice to Improve Supervision and Training. Londres, Reino Unido: Wiley publishers.

Safran, J. D. y Muran, J. C. (2005). La alianza terapéutica. Una guía para el tratamiento relacional. Bilbao, España: Desclée de Brower.

Skovnolt, T. M., Rennestad, M. H. y Jennings, L. (1997). Searching for Expertise in Counseling, Psychotherapy, and Professional Psychology. Educational Psychology Review, 9, 361-369. https://doi.or$\mathrm{g} / 10.1023 / \mathrm{A}: 1024798723295$

Stein, D. M. y Lambert, M. J. (1995). Graduate training in psychotherapy: Are trainingoutcomes enhanced? Journal of Consulting and Clinical Psychology, 63, 182-196.

Walfish, S., McAlister, B., O’Donnell, P. y Lambert, M. J. (2012). An Investigation of Self-Assessment Bias in Mental Health Providers. Psychological Reports, 110, 639-644. https://doi.org/10.2466/02.07.17. PR0.110.2.639-644

Wampold, B. E. y Brown, G. S. (2005). Estimating therapist variability: A naturalisticstudy of outcomes in managed care. Journal of Consulting and Clinical Psychology, 73, 914-923.

Watkins, C. E. (2011). Does Psychotherapy Supervision Contribute to Patient Outcomes? Considering Thirty Years of Research. The Clinical Supervisor, 30, 235-256. https://doi.org/10.1080/07325223.2011.619417.

Wilson, H. M., Davies, J. S. y Weatherhead, S. (2016). Trainee Therapists' Experiences of Supervision During Training: A Meta-synthesis. Clinical Psychology and Psychotherapy, 23, 340-351. https://doi.org/10.1002/ cpp.1957

Artículo recibido: 18/07/2019

Artículo aceptado: 20/10/2019 